\title{
AUTOMATED CALIBRATION TECHNIQUE FOR PROGRAMMABLE UNIVERSAL FREQUENCY-TO-DIGITAL CONVERTER
}

\author{
Sergey Y. Yurish ${ }^{1,2}$ and Nikolay V. Kirianaki ${ }^{2}$ \\ ${ }^{1}$ National University Lviv Polytechnic, Bandera str., 12, Lviv, UA \\ ${ }^{2}$ International Frequency Sensor Association (IFSA)
}

\begin{abstract}
The automated calibration technique for a trimming inaccuracy (calibration tolerance) of quartz resonator using in the universal precision frequency-to-digital converter is described in the paper. It was shown that such technique can be used also for reducing an error due to a quartz crystal aging and a temperature drift. The experimental technique and results for converter's error research in the temperature range $-40 \ldots+85{ }^{\circ} \mathrm{C}$ is also described in the paper. The aim of these experiments was to determine the margins of temperature range outside of which it is necessary to use the correction of frequency instability due to temperature changes. Copyright $($ C) 2006 IFAC
\end{abstract}

Keywords: calibration, oscillators, frequency conversion, frequency measurements, tolerance, quantization errors, converters

\section{INTRODUCTION}

Crystal oscillators are widely used in different measuring instruments and devices such as frequency counters, tachometers, counter/timers and frequencyto-digital converters, where an accurate frequency reference is required. Taking into account a high programmable accuracy (up to $0.001 \%$ ) of the developed Universal Frequency-to-Digital Converter (UFDC-1) described by Yurish, et al., (2005), it needs a very accurate frequency standard at least $\leq 0,0001 \%$. Such relative error has a temperature compensated crystal oscillator (TCXO) or oven controlled crystal oscillator. However, these oscillators are relatively expensive devices and its price is higher than the UFDC-1 price. In addition, the oven controlled crystal oscillator can not be used in embedded applications as digital or smart microelectronic sensors.

A crystal oscillator is a relatively inexpensive method of obtaining an accurate frequency standard if it will be periodically calibrated. The frequency or rate of vibration of quartz oscillator is determined by the cut, size, shape of the resonator, etc. Most commonly used are slices cut under an angle of $35^{\circ}$ $15^{\prime}$ with the $Z$ axis (the AT cut). These AT-cut slices can be used for frequencies from $0.5 \mathrm{MHz}$ to 350 $\mathrm{MHz}$. The primary frequency determining factor for AT cut is thickness since they vibrate in the thickness shear mode. The precision with which the thickness is controlled determines the variation from crystal to crystal from a nominal center frequency.

The temperature characteristics of AT-cuts crystals are described by a $3^{\text {rd }}$ order parabola. It is then possible to describe the relative change of frequency:

$$
\frac{\Delta f}{f}=A_{i} \Delta T+C_{i} \Delta T^{3},
$$

where $\Delta T=T-T_{i}$ and $T_{i}$ is the inflection temperature; $A_{i}$ is the coefficient, which is depend on the quartz crystal construction and determined by the angle of cut; $C_{i}$ is the constant; $T_{i}$ is varied in the range from +25 to $+35{ }^{\circ} \mathrm{C}$ for AT-cut depend on the crystal size (Plons'kiy, 1966 and Al'tshuller, 1975).

The frequency variation due to adjustment inaccuracy for a room temperature crystal oscillator (RTXO) can exceed $30 \mathrm{ppm}$ (or $0.003 \%$ ) and has a systematic character. For example, $16 \mathrm{MHz}$ crystal oscillators from Siward with $30 \mathrm{ppm}$ determined 
tolerance has the real frequency $16001400 \mathrm{~Hz}$ that corresponds to $90 \mathrm{ppm}(0.009 \%)$ reference error.

In order to use the UFDC-1 with low cost crystal oscillators for conversions with quantization error better than $0.01 \%$ it is expediently to make a calibration with the aim to compensate the adjustment or trimming inaccuracy. In other case, the calibration is not necessary.

\section{THE UFDC-1 AUTOMETAD CALIBRATION}

The calibration should be made in real working conditions with the use of recommended $16 \mathrm{MHz}$ crystal oscillator. At this calibration procedure, the converter should be connected to PC through the serial interface RS-232. Any terminal software can be used for this purpose, for example, Terminal $\mathrm{V} 1.9 \mathrm{~b}$ for Windows.

The calibration sequence for the UFDC-1 is the following:

1) The test command "T" toggles the UFDC-1 in the calibration mode. The sequence of rectangular pulses with frequency $f_{\text {osd }} / 2$ is generated on its TEST output. 2) The frequency of this pulse sequence should be measured with the help of any external frequency counter with accuracy not worse than $0.0001 \%$ or at least $0.0005 \%$.

3) The fractional part of measuring result is rejected and the number $8000000(\mathrm{~Hz})$ should be subtracted from the integer part.

4) The received correction factor $\Delta$ need to be converted into the hexadecimal number.

5) Use the command $" F \pm \Delta "$ (with taking into account the result sign) to put the correction value into the UFDC-1. The command "F" without parameters indicates the internal value of correction factor and can be used for checking the loaded number.

The calibration is performed only once. The correction factor $\Delta$ is stored in the nonvolatile memory of the UFDC-1, which holds this value after power-off.

\section{Example:}

Toggle the UFDC-1 in the calibration mode by "T" command entered in any terminal software, working under Windows operation systems:

$>\mathrm{T}$

Let the measured frequency on the TEST output is $8000694.257865 \mathrm{~Hz}$, for example. We reject a fractional part and received the integer number is $8000694 \mathrm{~Hz}$. Then we calculate the correction factor $8000694-8000000=694 \mathrm{~Hz}$ and convert the result into the hexadecimal number $(694)_{10}=$ (2B6) 16 . Finally, we send the correction command with taking into account the correction factor's sign: $>\mathrm{F}+2 \mathrm{~B} 6$

Then to check programmed value we send the command:
The returned result 2B6 testifies about the properly stored value of correction factor for the given quartz crystal. After that, the UFDC-1 is ready for conversion in the frame of programmable accuracy ranges from 1 to $0.001 \%$.

If the primary frequency of crystal oscillator is $16000937.5 \mathrm{~Hz}$ and the converted frequency $f_{x}=25000 \mathrm{~Hz}$, the result without calibration $(\Delta=0)$ and with specified quantization error $\delta_{q \text { setup }}=0.001 \%$ would be $f_{x i}=24998.0002 \mathrm{~Hz}$. This corresponds to the conversion error $\delta_{q}=0.008 \%>\delta_{q \text { setup }}$. After calibration, the measured frequency is $f_{x i}=25000.1781 \mathrm{~Hz}$. It corresponds to the relative error $\delta_{q}=0.0007 \%<\delta_{q \text { setup }}$

Besides the trimming inaccuracy, the primary frequency of crystal oscillator is also influenced by different drifts due to temperature changes, aging, supply-voltage changes and other environmental changes. The periodical calibration is also useful for elimination of aging influence as well as temperature changes.

Taking into account the industrial working temperature range for the UFDC- $1\left(-40^{\circ} \mathrm{C} \ldots+85^{\circ} \mathrm{C}\right)$ it is also expediently to investigate the conversion error in this temperature range, a possibility for temperature drift calibration and temperature margins in which the calibration will be necessary.

\section{TEMPERATURE DEPENDENCE OF CONVERSION ERROR}

\subsection{Objective of experiments, experimental setup and technique}

The main objective of experiments is to investigate the temperature dependence of conversion error in all working temperature range $\left(-40^{\circ} \mathrm{C} \ldots+85^{\circ} \mathrm{C}\right)$ and determinate the margins where it will be necessary to correct the temperature drift.

The experimental research was carry out in the climatic chamber of Technical University of Catalonia (UPC Barcelona, Spain). The UFDC-1 evaluation kit circuit diagram is shown in Fig.1.

A data acquisition, result indication and communication between the UFDC-1 and PC were made in LabView 7.0 environment. An appropriate virtual instrument's front panel is shown in Fig. 2. It consists of four main modules: serial port configuration, measuring modes and conversion error setup, results indication and statistical calculations, and calibration modules. The last one lets input the correction factor $\Delta$ and its sign. The virtual instrument's block diagram (measuring mode setup, indication and calculation modules) is shown in Fig.3, and port configuration module - in Fig.4. 


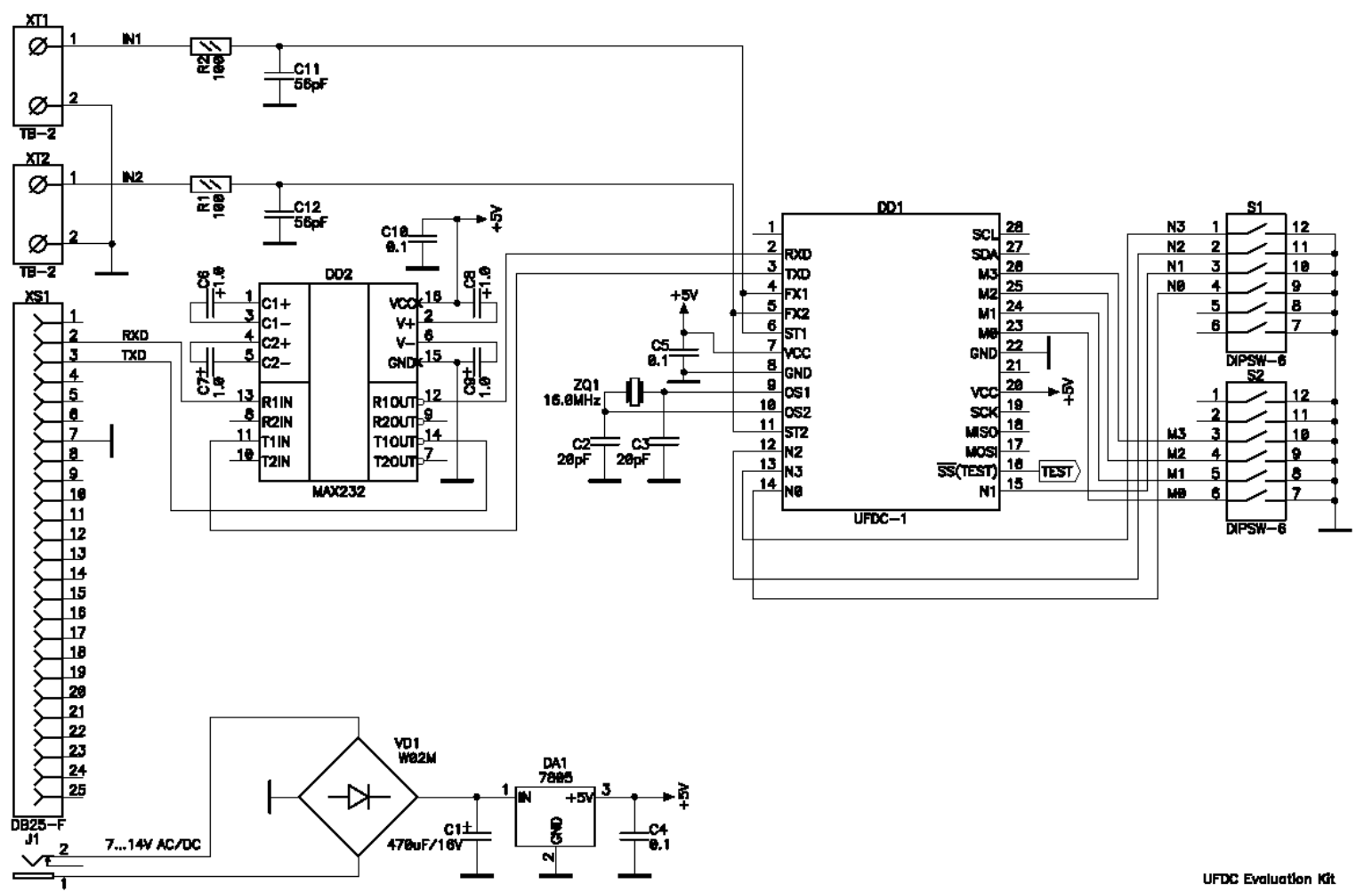

Fig. 1. UFDC-1 evaluation kit circuit diagram.

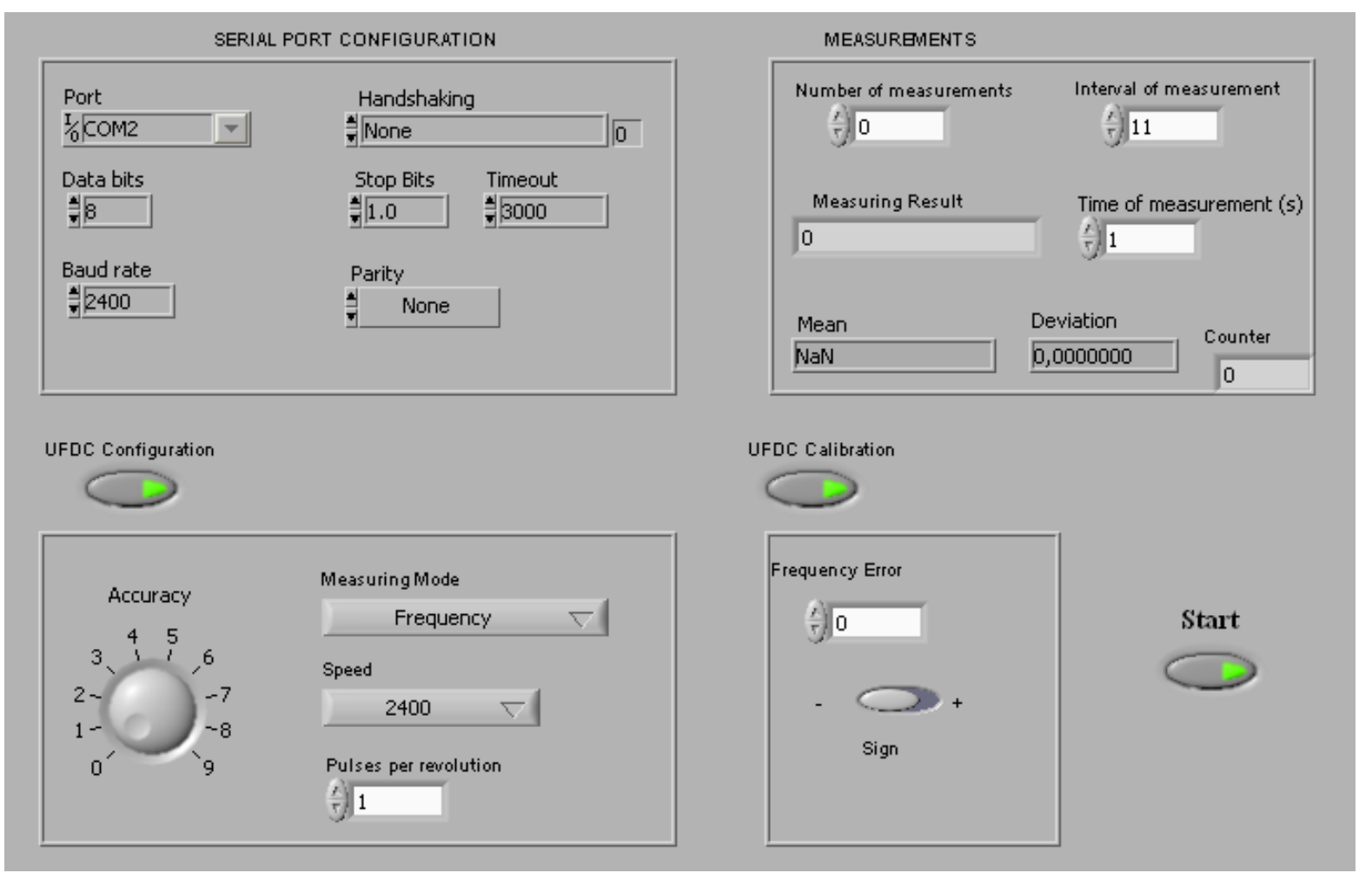

Fig. 2. Virtual instrument's front panel for the UFDC-1 experimental research. 


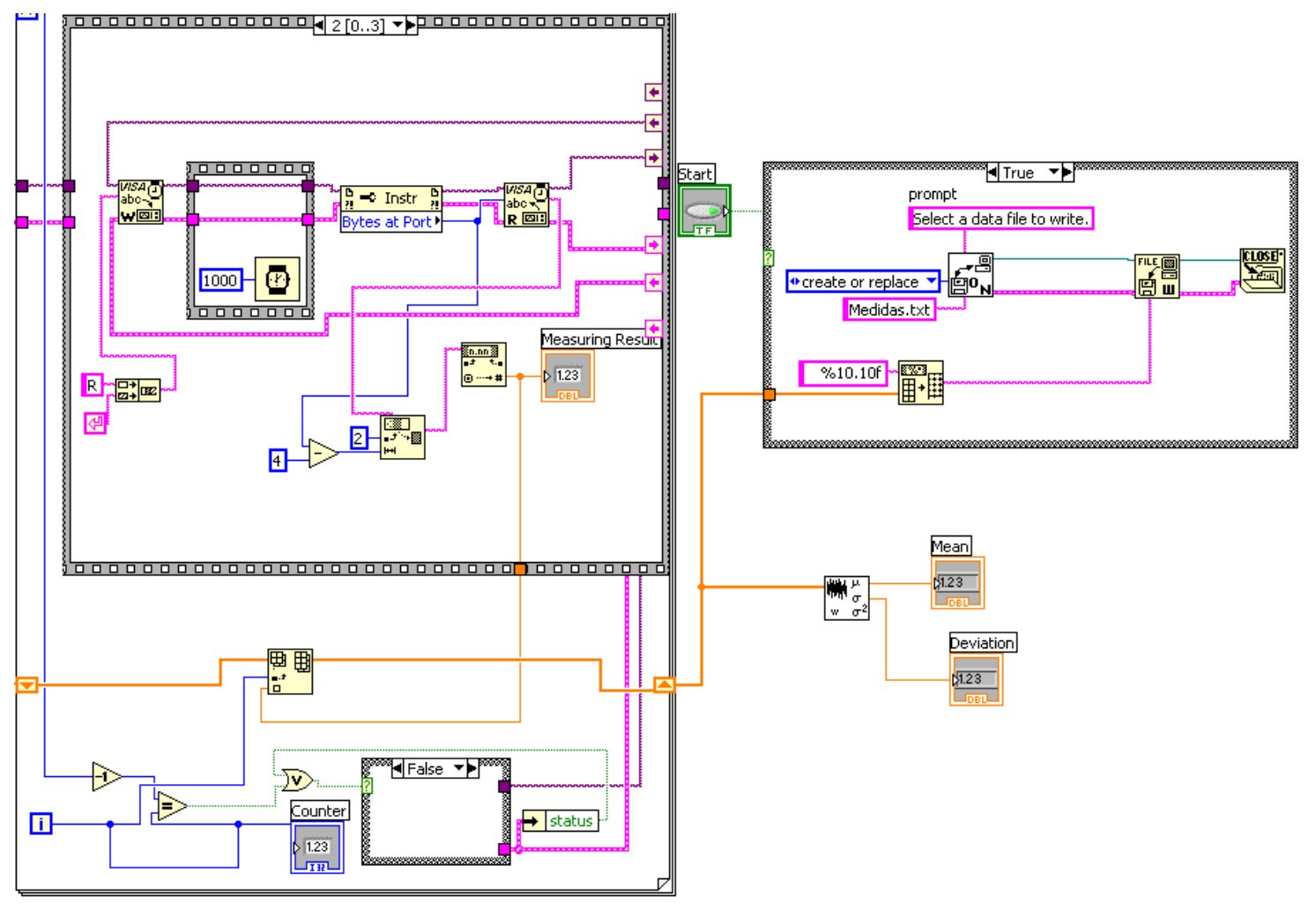

Fig. 3. Block diagram of measuring mode setup, calculation and results indication modules.

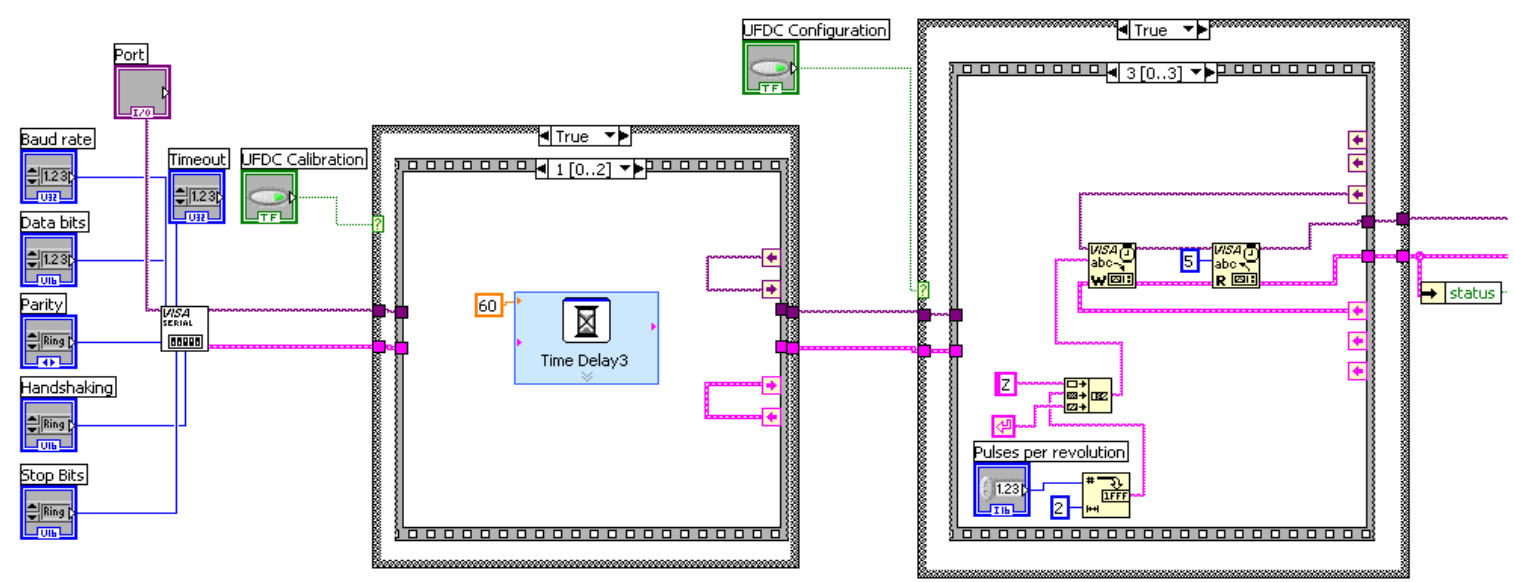

Fig. 4 Block diagram of serial port configuration module.

The experimental setup is shown in Fig. 5.

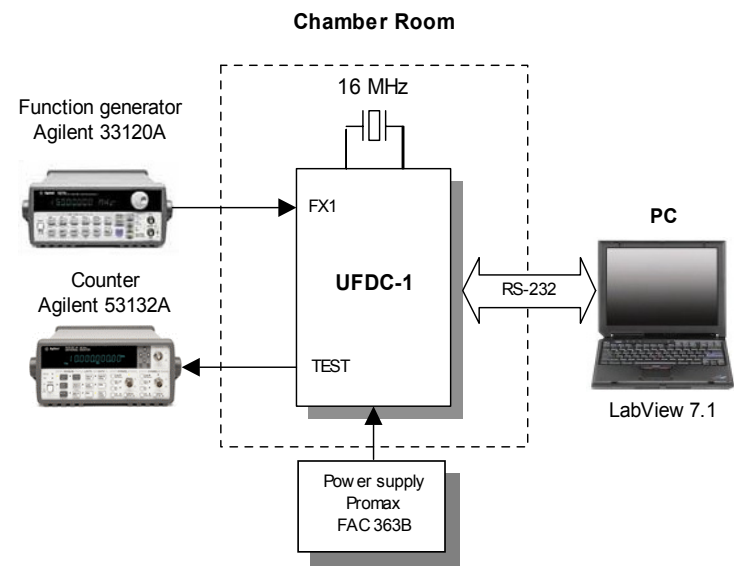

Fig. 5 Experimental setup.
After 45 minutes temperature stabilization the UFDC- 1 was calibrated at $+21^{\circ} \mathrm{C}$. The quantization error was chosen $0.001 \%$ and frequencies $1 \mathrm{~Hz}, 1 \mathrm{kHz}$ and $1 \mathrm{MHz}$ were measured one hundred times of each through $2 \mathrm{~s}$ time interval. The investigated temperature range was $-40{ }^{\circ} \mathrm{C} \ldots+80{ }^{\circ} \mathrm{C}$ with $10{ }^{\circ} \mathrm{C}$ step at $50 \%$ relative humidity. The input signal of appropriate frequency was generated by the function generator Agilent 33120A. The frequency counter Agilent 53132A was used for frequency measurement at the UFDC's TEST output.

\subsection{Experimental results}

Frequency vs temperature characteristics for $1 \mathrm{~Hz}$, $1 \mathrm{kHz}$ and $1 \mathrm{MHz}$ are shown in Table 1 and in Fig. 6 a-c accordingly. 
Table 1 Experimental results: frequency vs temperature (data in frames correspond to the ranges where correction does not need)

\begin{tabular}{|c|c|c|c|c|c|c|}
\hline & \multicolumn{2}{|c|}{$1 \mathrm{~Hz}$} & \multicolumn{2}{|c|}{$1 \mathrm{kHz}$} & \multicolumn{2}{|c|}{$1 \mathrm{MHz}$} \\
\hline $\mathrm{t},{ }^{\circ} \mathrm{C}$ & $\mathbf{f}_{\mathrm{x} \text { mean }}, \mathrm{Hz}$ & $|\Delta \mathrm{f}|, \mathrm{Hz}$ & $\mathbf{f}_{\mathrm{x} \text { mean }}, \mathrm{Hz}$ & $|\Delta \mathrm{f}|, \mathrm{Hz}$ & $\mathbf{f}_{\mathrm{x} \text { mean }}, \mathrm{Hz}$ & $|\Delta \mathrm{f}|, \mathrm{Hz}$ \\
\hline-40 & 0,99998840381 & 0,00001159619 & 999,989301511 & 0,010698488 & 999989,883188 & 10,1168118 \\
\hline-30 & 0,99997040622 & 0,00002959378 & 999,980002999 & 0,019997000 & 999979,581952 & 20,4180470 \\
\hline-20 & 0,99997516445 & 0,00002483555 & 999,979203143 & 0,020796856 & 999979,881988 & 20,1180110 \\
\hline-10 & 0,99997804490 & 0,0000219551 & 999,980002999 & 0,019997000 & 999984,582552 & 15,4174478 \\
\hline 0 & 0,99998572280 & 0,0000142772 & 999,986014637 & 0,013985362 & 999989,183104 & 10,8168958 \\
\hline+10 & 0,99998984286 & 0,00001015714 & 999,998800167 & 0,001199832 & 999998,484220 & 1,5157798 \\
\hline+20 & 0,99998966262 & 0,00001033738 & 1000,000000000 & 0,000000000 & 1000002,634718 & 2,6347180 \\
\hline+30 & 1,00000342059 & 0,00000342059 & 1000,009998800 & 0,009998800 & 1000009,185504 & 9,1855041 \\
\hline+40 & 1,00001093949 & 0,00001093949 & 1000,009998800 & 0,009998800 & 1000016,636398 & 16,63639813 \\
\hline+50 & 1,00001253972 & 0,00001253972 & 1000,019197880 & 0,019197880 & 1000024,087292 & 24,08729212 \\
\hline+60 & 1,00002213831 & 0,00002213831 & 1000,029497040 & 0,029497040 & 1000029,287916 & 29,28791612 \\
\hline+70 & 1,00002811700 & 0,00002811700 & 1000,029997000 & 0,029997000 & 1000032,038246 & 32,03824611 \\
\hline+80 & 1,00002355818 & 0,00002355818 & 1000,029997000 & 0,029997000 & 1000032,288276 & 32,28827611 \\
\hline
\end{tabular}

$1 \mathrm{~Hz}$

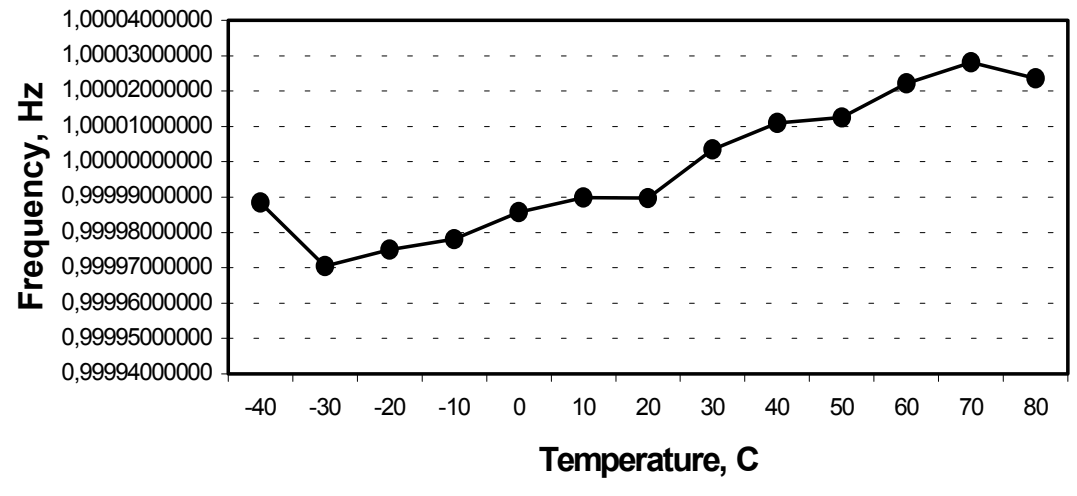

a)

$1 \mathrm{kHz}$

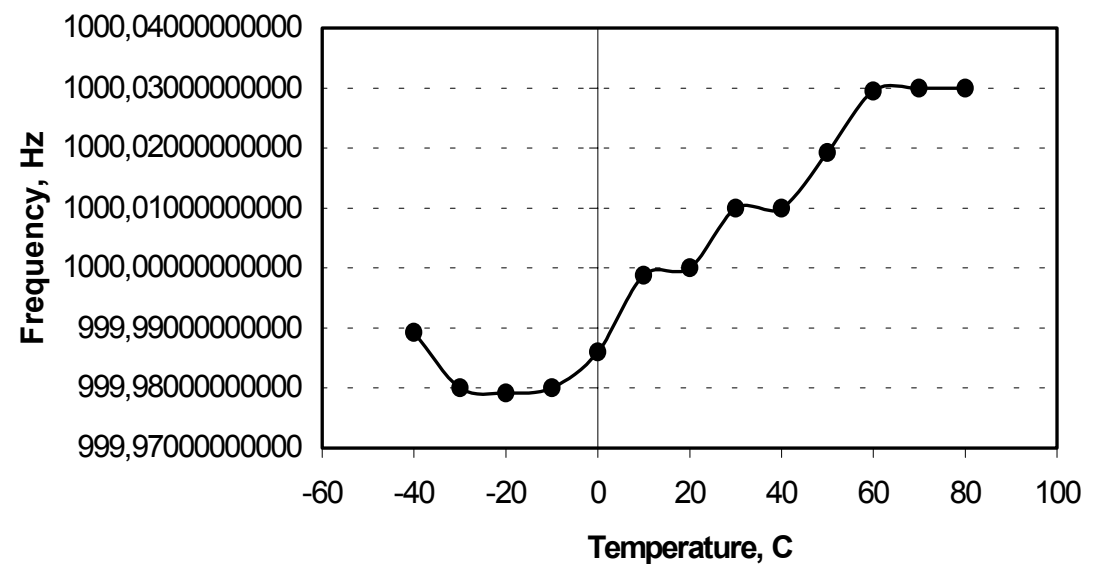

$1 \mathrm{MHz}$

b)

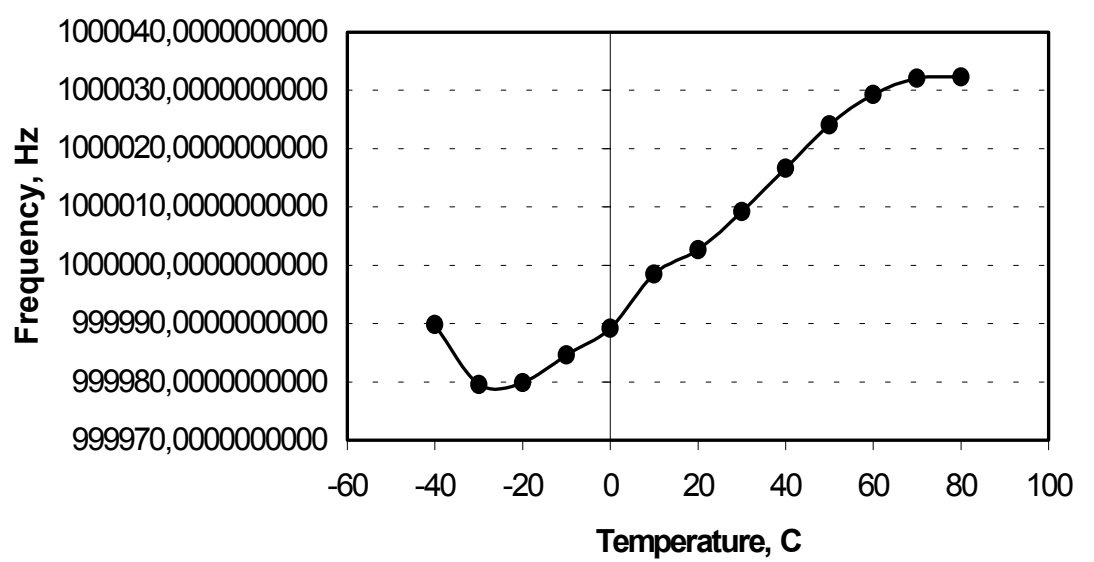

Fig. 6. Frequency vs temperature characteristics for $1 \mathrm{~Hz}$ (a), $1 \mathrm{kHz}$ (b) and $1 \mathrm{MHz}$ (c) 
Due to temperature changes, the conversion error at $1 \mathrm{~Hz}$ frequency measurement can be increased up to $0.003 \%$ (or $30 \mathrm{ppm}$ ). It is true for temperature ranges $-30 \ldots 0{ }^{\circ} \mathrm{C}$ and $+50 \ldots+80{ }^{\circ} \mathrm{C}$. In the rest part of temperature range, the conversion error does not exceed the programmed value $0.001 \%$ (or $10 \mathrm{ppm}$ ).

At $1 \mathrm{kHz}$ frequency measurement the conversion error also can be $30 \mathrm{ppm}$ and its value exceeds the programmed error in the temperature ranges $-30 \ldots 0{ }^{\circ} \mathrm{C}$ and $+40 \ldots+80{ }^{\circ} \mathrm{C}$.

Analogically, at $1 \mathrm{MHz}$ frequency measurement the conversion error is $32 \mathrm{ppm}$ in the temperature ranges $-30 \ldots+10{ }^{\circ} \mathrm{C}$ and $+30 \ldots+80{ }^{\circ} \mathrm{C}$.

Fig. 5 shows the temperature intervals in which necessary to calibrate the UFDC- 1 .

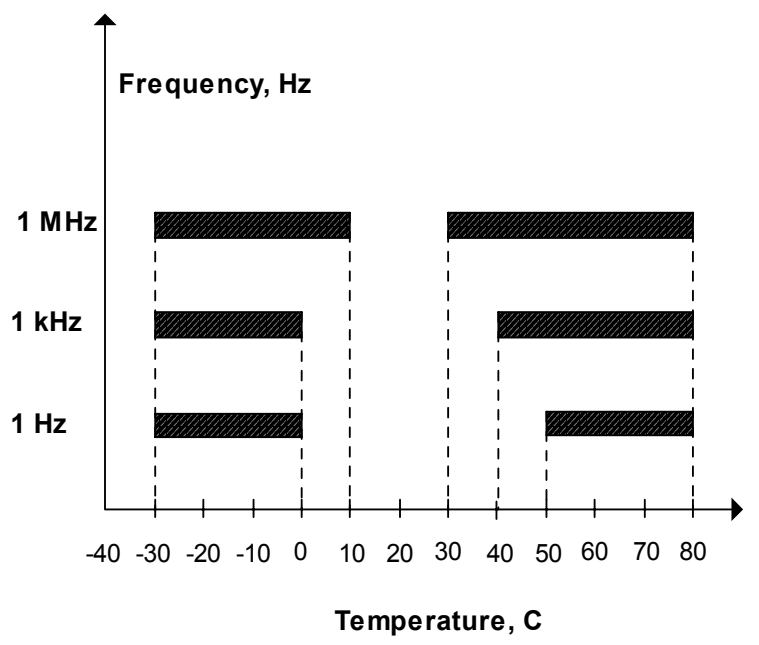

Fig.5. Temperature intervals in which it is necessary to calibrate the UFDC-1.

Obtained experimental results coincide with the temperature-frequency characteristic for standard quartz oscillator of RTXO type with the AT-cut, described by a $3^{\text {rd }}$ order parabola, Gufflet (2003). Such quartz resonators have minimal temperature changes in the temperature range of $0 \ldots+50{ }^{\circ} \mathrm{C}$. At frequency increasing, the temperature range is narrowed, probably, because of strong influence of short-term instability at high frequency measurements.

At frequency measurement in temperature range near $-40{ }^{\circ} \mathrm{C}$ no any correction is necessary. It is explained by the temperature-frequency characteristic of this type of quartz oscillators.

If the UFDC-1 is working outside of experimental determined temperature ranges with the quantization error better then $0.01 \%$, the correction procedure described above should be used. If due to some reasons the correction is not possible, it is expediently to use a TXCO type quartz crystal with the frequency variation not more than $5 \mathrm{ppm}$ in the working temperature range $-20 \ldots+70{ }^{\circ} \mathrm{C}$. Besides, the UFDC-1 can work well also with an external precise quartz generator.

\section{CONCLUSIONS}

The main contribution to the frequency reference error contributes the trimming inaccuracy (or calibration tolerance). This component has a systematic character. If the necessary quantization error of the UFDC- 1 must be better than $0.01 \%$, the correction procedure should be used. It lets to the UFDC-1 work well with a low-cost RTXO quartz oscillator. It is a very important property for different embedded applications, for example, digital and smart microelectronic sensors.

Besides, the proposed correction technique lets also to reduce an aging error component as well as the frequency instability due to temperature drift in the wide temperature range. Experimental research has determined the margins for the working temperature range $-40 \ldots+85{ }^{\circ} \mathrm{C}$ in frame of which the correction is necessary.

The further research consists in development of automatic calibration procedure for trimming inaccuracy that will take into account also the fractional part of frequency value. It will let to decrease the conversion error of UFDC-1 up to $0,0001 \%$ or better.

\section{ACKNOWLEDGEMENTS}

The authors appreciate the technical support of Mr. Francis Lopez from Technical University of Catalonia (UPC, Barcelona, Spain) in the experimental setting. This work has been funded by the Spanish Ministry of Education and Science, project DPI2002-00707 and grant SAB2002-0062.

\section{REFERENCES}

Al'tshuller, G.B. (1975). Frequency control in quartz generators. Svyaz, Moscow. (in Russian).

Gufflet, Dr. N. (2003). Quartz crystal resonators, brief overview. KVG Quartz Crystal Technology. http://www.kvg-gmbh.de

Plons'kiy, O.P. (1966). Quartz generators on transistors. Tekhnika, Kyjv. (in Ukrainian).

Yurish, S. Y., N.V. Kirianaki and R. Pallàs-Areny (2005). Universal Frequency-to-Digital Converter for Quasi-Digital and Smart Sensors: Specifications and Applications. Sensor Review, 25, 92-99. 